\title{
Wiping Systems: Influence of the Surface Tension in Water Film Rupture ${ }^{\dagger}$
}

\author{
Celia Miguel *, Manuel García and Bruno Pereiras \\ Área de Mecánica de Fluidos, Universidad de Oviedo, Gijón 33203, Spain; garciadmanuel@uniovi.es (M.G.); \\ pereirasbruno@uniovi.es (B.P.) \\ * Correspondence: celiamiguelg@gmail.com; Tel.: +34-985-18-26-61 \\ † Presented at the 2nd International Research Conference on Sustainable Energy, Engineering, Materials and \\ Environment (IRCSEEME), Mieres, Spain, 25-27 September 2018.
}

Published: 7 November 2018

\begin{abstract}
Wiping system are widely employed in industrial processes to remove liquid residue from the final product. Therefore, the performance of the impingement of a gas jet upon a liquid surface or molten bath is relevant in many industrial and metallurgical processes. This work is focused on wiping systems used in steel cold rolling, where a system composed of air jets impinging on the steel strip should remove the thin film of lubricant emulsion coming from the rolling bite. In the bibliography there are references of analytical cases of reduction of coating thickness by means of an air jet, such as galvanizing processes, which do not take into account surface tension. In the case of drying systems film rupture exists. A lot of variables are implied in the film rupture theory. Upon all of them the most important variable is the surface tension. The aim of this work is to study the influence of surface tension in film rupture. For this reason, a model of a jet wiping a film of water upon a strip is developed with and without surface tension.
\end{abstract}

Keywords: surface tension; wiping; film rupture; CFD

\section{Introduction}

A high drying efficiency of the final product is essential for many steelmaking processes in order to obtain the quality required by customers. Many devices, commonly found in tin plate or cold rolling lines, are based on high velocity jets which wipe the liquid from the surface. The effectiveness of the drying depends on the jets configuration: size of the nozzle, distance to the strip and angle of blowing. In general, the liquid is in thin-film shape which make the problem attractive to be solved analytically; in fact, there are models of liquid films through a steel strip for different steelmaking processes.

In the bibliography there are many references to the analytical model that relates pressure with final thickness. Thornton and Graff [1] developed an analytical model for a hot dip galvanizing process. Ellen and $\mathrm{Tu}$ [2], made a better approximation by including in the formula of Thornton and Graff the shear stress by means of a boundary condition.

However, most of such models are restricted to a high viscous fluid, and without film rupture which drives to not discard the surface tension effect. In case of wiping processes, film rupture has to be taken into account, so it is necessary to take into account the surface tension in order to achieve the solution of the problem. Moriarty, Schwartz and Tuck [3] develop an analytical model of a drop of water wiped horizontally by means of an air jet, taking into account the surface tension.

The objectives of the present study are to study the effects of the surface tension in the resolution of a wiping problem and to analyze the 3D effects of the air jet. For this purpose, a CFD model in 
ANSYS FLUENT was developed in order to evaluate the behavior of a water film on a horizontal continuous strip being blown by a pressure jet.

\section{Numerical Model}

The simulation of the model was made in ANSYS FLUENT 16.0, which uses the finite volume method in order to resolve the Navier-Stokes equations. The mesh (Figure 1), built in ANSYS ICEM $\mathrm{CFD}$, is structured and composed of $1 \mathrm{M}$ hexahedrical cells. Mesh quality, assessed by the determinant of the jacobian matrix (Determinant $3 \times 3 \times 3$ ), is above 0.7 .

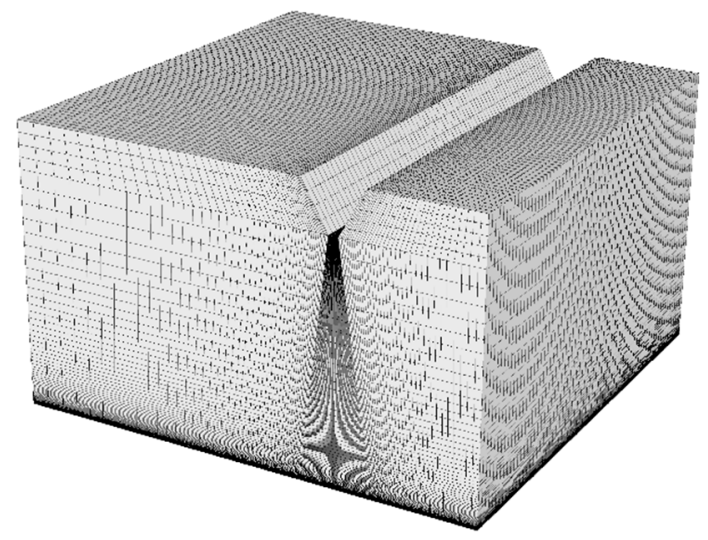

Figure 1. General view of the mesh.

For the resolution of the multiphase model, the VOF (Volume Of Fluid) model has been selected, being the interface model set as "sharp". This method has been validated experimentally by several authors in galvanizing models [4].

The simulation was carried out in transient regime since the process in completely unsteady, although the boundary conditions remain steady during each simulation. The temporal term is approximated by an implicit first order scheme. The velocity-pressure pair was reproduced through a SIMPLE algorithm. The upwind scheme has been used for the discretization of the convective terms, while classical approximation for centered references has been used for the diffusive terms. The time step was set at $10^{-4}$ and the residuals was set at $10^{-5}$. The choice of turbulence model has been based on numerous papers [5,6], where the RNG k- $\varepsilon$ combined to "Non-equilibrium Wall Functions" is strongly recommended. The input thickness is set by the modification of the input conditions, more exactly with the velocity inlet. The details of the boundary conditions can be seen in Figure 2 .

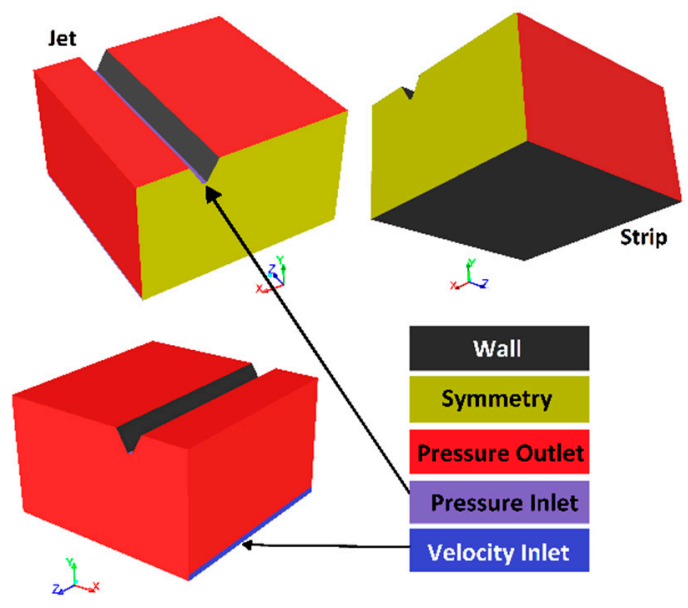

Figure 2. Geometry and boundary conditions. 
Eight simulations were made with surface tension and eight simulations without surface tension for an initial thickness of water layer of $2 \mathrm{~mm}$. In the same way, six simulations are carried out with and without surface tension for an initial thickness of $0.1 \mathrm{~mm}$. In total, 28 simulations are carried out (Table 1).

Table 1. Simulations.

\begin{tabular}{|c|c|}
\hline \multicolumn{2}{|c|}{ With/Without Surface Tension } \\
\hline $\mathbf{t}=\mathbf{2} \mathbf{~ m m}$ & $\mathbf{t}=0.1 \mathrm{~mm}$ \\
\hline Air Pressure [Pa] & Air Pressure [Pa] \\
\hline 22,000 & 5000 \\
\hline 33,000 & 10,000 \\
\hline 60,000 & 22,000 \\
\hline 100,000 & 33,000 \\
\hline 150,000 & 60,000 \\
\hline 221,000 & 100,000 \\
\hline 300,000 & \multicolumn{1}{|c}{} \\
\hline 350,000 & \multicolumn{1}{|c}{} \\
\hline
\end{tabular}

\section{Analysis of the Results and Discussion}

\subsection{Average Thickness of Water at the Outlet}

In order to perform a first analysis of the influence of the surface tension, the average final thickness is obtained for different jets pressures that are shown in the Figure 3. The arithmetic average of the 500 data taken from the model was made in order to obtain these average thickness. That is translated into $0.05 \mathrm{~s}$ of simulation, or $0.65 \mathrm{~m}$ of strip. As can be seen in Figure 4, surface tension appears to have no effect on the average thickness which remain after the nozzle wiping.

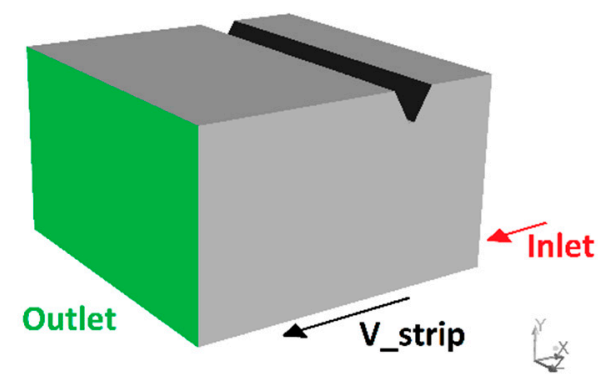

Figure 3. Outlet and inlet of the flow in the simulation domain.
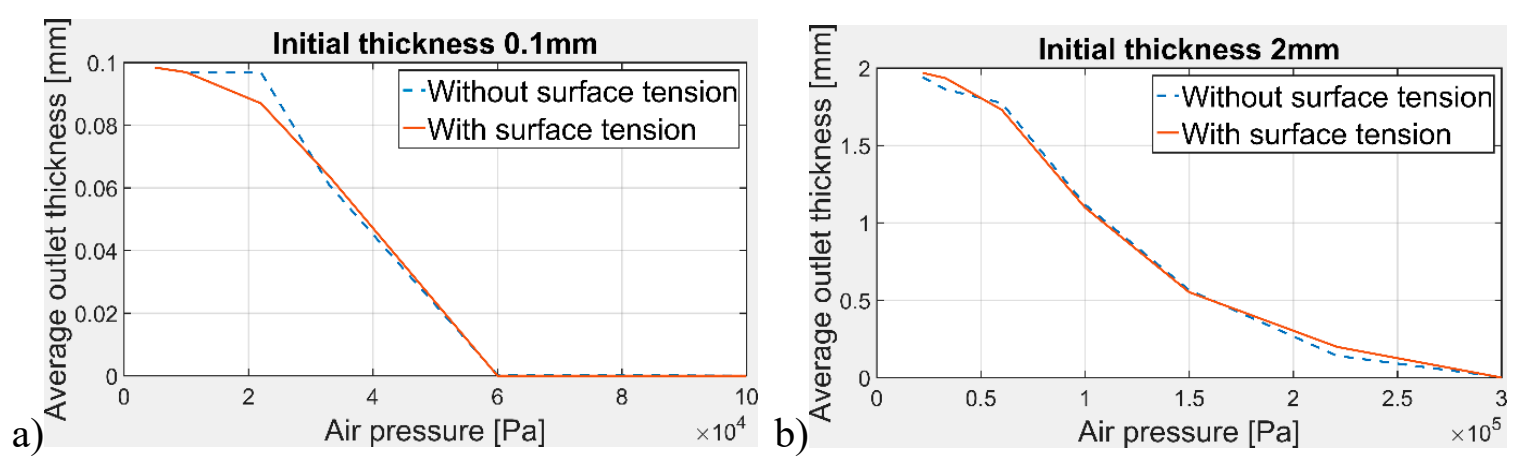

Figure 4. Average thickness at the outlet for initial thickness 0.01 (a) $\mathrm{mm}$ and $2 \mathrm{~mm}$ (b). 


\subsection{Waves Analysis}

In order to know the performance of the layer of water at the outlet of the domain, the outlet thickness is shown as a function of time, Figure 5. This signal is processed by FFt techniques (Figure 6) in order to identify presence of waves of ordered structure on the surface of the coating.

In the Figure 6 at the right, there is a dominant frequency of $100 \mathrm{~Hz}$. This frequency can be related with the wavelength $(\lambda)$ of the wave of water by means of the following equation:

$$
\lambda=V o / f
$$

Where $V o$ is the velocity of the strip $(13 \mathrm{~m} / \mathrm{s})$, and $f$ is the freciency of the peack of the spectrum. For the case with surface tension, the wavelength is $0.13 \mathrm{~m}$. This wavelength can be easily appreciated in the plot that relates outlet thickness in function of time (Figure 5).

In the same way, the dominant frequency of the case without surface tension (Figure 6) is $40 \mathrm{~Hz}$. The wavelength is equal to $0.33 \mathrm{~m}$ (Figure 5). The generation of this waves is related with the turbulence of the air jet and the vortex detachment.
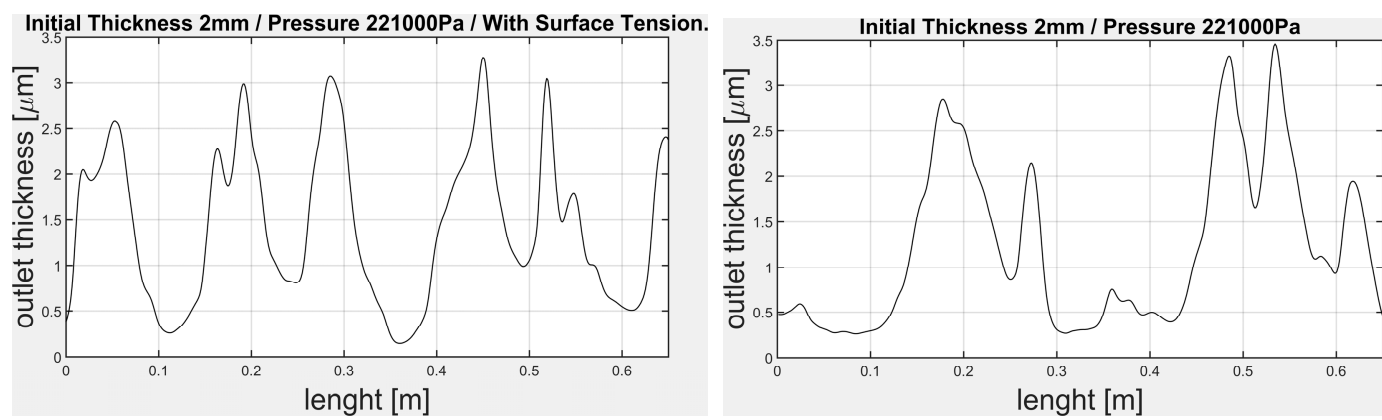

Figure 5. Thickness vs. distance ((Left) with surface tension, (Right) without surface).
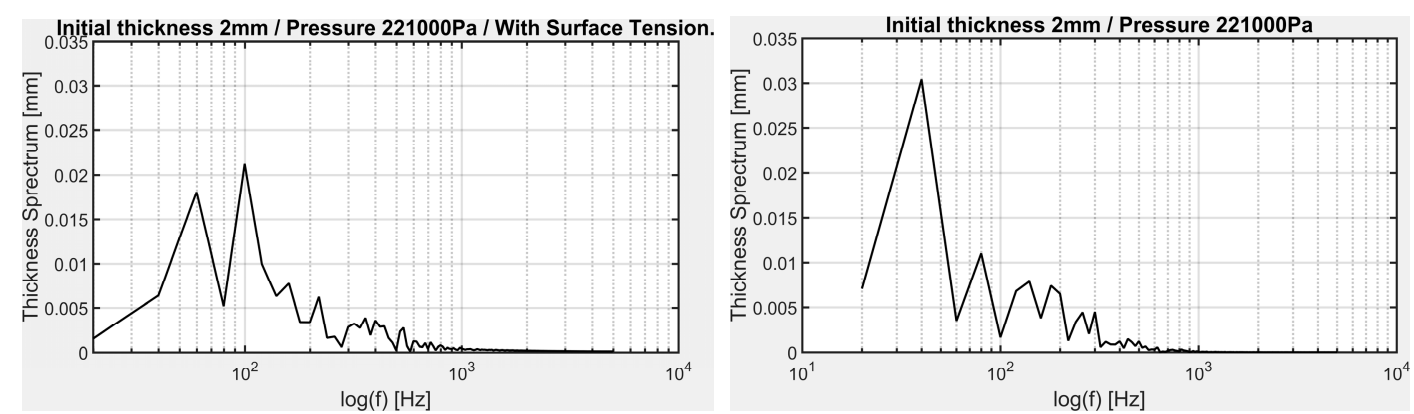

Figure 6. Thickness spectrum ((Left) with surface tension, (Right) without surface).

\section{Conclusions}

In the elaboration of this work, the following conclusion were reached: The effect of the surface tension does not affect in an evident way the final thickness of the resulting layer after the sweep and using spectral analysis, we can know if there are waves or not and know their wavelength.

\section{References}

1. Thornton, J.A.; Graff, H.F. An analytical description of the jet finishing process for hot-dip metallic coatings on strip. Metall. Trans. B 1976, 7, 607-618.

2. Ellen, C.H.; Tu, C.V. An Analysis of Jet Stripping of Molten Metallic Coatings. In Proceedings of the Eighth Australasian Fluid Mechanics Conference, Newcastle, New South Wales, 28 November-2 December 1983; pp. 2C.4-2C.7.

3. Moriarty, J.A.; Schwartz, L.W.; Tuck, E.O. Unsteady spreading of thin liquid films with small surface tension. Phys. Fluids A 1991, 3, 733-742. 
4. Pfeiler, C.; Mataln, M.; Kharicha, A.; Riener, C.K.; Angeli, G. Importance of the Zinc Film Modeling for Gas Jet Wiping Simulations. In Proceedings of the International Conference on Zinc and Zinc Alloy Coated Steel Sheet (Galvatech 2015), Toronto, ON, Canada, 31 May-4 June 2015; pp. 1-15.

5. Elsaadawy, E.A.; Hanumanth, G.S.; Balthazaar, A.K.S.; McDermid, J.R.; Hrymak, A.N.; Forbes, J.F. Coating weight model for the continuous hot-dip galvanizing process. Metall. Mater. Trans. B 2007, 38, 413-424.

6. Tamadonfar, P. Numerical Investigation of Multiple-Impinging Slot Jets in the Gas-Jet Wiping of Liquid Zinc Coatings. Ph.D. Thesis, McMaster University, Hamilton, Canada 2010.

(C) 2018 by the authors. Licensee MDPI, Basel, Switzerland. This article is an open access article distributed under the terms and conditions of the Creative Commons Attribution (CC BY) license (http://creativecommons.org/licenses/by/4.0/). 